University of Texas at El Paso

ScholarWorks@UTEP

\title{
Interval Computations as a Particular Case of a General Scheme Involving Classes of Probability Distributions
}

\author{
Scott Ferson \\ Lev Ginzburg \\ Vladik Kreinovich \\ The University of Texas at El Paso, vladik@utep.edu \\ Harry Schulte
}

Follow this and additional works at: https://scholarworks.utep.edu/cs_techrep

Part of the Computer Engineering Commons

Comments:

UTEP-CS-99-36b.

Published in: Juergen Wolff von Gudenberg and Walter Kraemer (eds.), Scientific Computing, Validated Numerics, Interval Methods, Kluwer, Dordrecht, 2001, pp. 355-366.

\section{Recommended Citation}

Ferson, Scott; Ginzburg, Lev; Kreinovich, Vladik; and Schulte, Harry, "Interval Computations as a Particular Case of a General Scheme Involving Classes of Probability Distributions" (2001). Departmental Technical Reports (CS). 575.

https://scholarworks.utep.edu/cs_techrep/575

This Article is brought to you for free and open access by the Computer Science at ScholarWorks@UTEP. It has been accepted for inclusion in Departmental Technical Reports (CS) by an authorized administrator of ScholarWorks@UTEP.For more information, please contact Iweber@utep.edu. 


\title{
INTERVAL COMPUTATIONS AS A PARTICULAR CASE OF A GENERAL SCHEME INVOLVING CLASSES OF PROBABILITY DISTRIBUTIONS
}

\author{
Scott Ferson and Lev Ginzburg \\ Applied Biomathematics \\ 100 North Country Road, Setauket, NY 11733, USA \\ scott@ramas.com \\ Vladik Kreinovich ${ }^{a}$ and Harry Schulte ${ }^{b}$ \\ ${ }^{a}$ Computer Science Department \\ ${ }^{b}$ Digital Media Center \\ University of Texas, El Paso, TX 79968, USA \\ vladik@cs.utep.edu, hschulte@utep.edu
}

Keywords: interval computations, probability distributions

\begin{abstract}
Traditionally, in science and engineering, measurement uncertainty is characterized by a probability distribution; however, often, we don't know this probability distribution exactly, so we must consider classes of possible probability distributions. Interval computations deal with a very specific type of such classes: classes of all distributions which are located on a given interval. We show that in general, we need all closed convex classes of probability distributions.
\end{abstract}

\section{INTRODUCTION}

Even after the most accurate measurement, there is still some uncertainty about the actual value of the measured quantity. Traditionally, this uncertainty is characterized by a probability distribution $P$ on the set $X$ of possible values of the measured quantity. However, often, we do not know $P$ exactly - because several different probability measures are consistent with all the measurements. We must therefore consider 
the class $\mathcal{P}$ of all probability measures which are consistent with measurements. So, the general uncertainty can be described by a class of probability distributions. For example, an interval uncertainty (when $x \in[a, b]$ is the only available information) corresponds to the class $\mathcal{F}_{[a, b]}$ of all probability distributions $P$ located on a given interval $[a, b]$.

In many real-life situations, it is difficult or even impossible to directly measure the value of the quantity $y$ in which we are interested. In such situations, we can often determine the value of this quantity indirectly: namely,

- we measure the quantities $x_{1}, \ldots, x_{n}$ which are related to $y$ and which can be measured directly, and then,

- we use the known relationship $y=f\left(x_{1}, \ldots, x_{n}\right)$ between $x_{i}$ and $y$ to estimate the value of $y$ based on the measured values $\widetilde{x}_{i}$ of $x_{i}$ : $\widetilde{y}=f\left(\widetilde{x}_{1}, \ldots, \widetilde{x}_{n}\right)$.

This is called data processing, or indirect measurement. We know the uncertainty class $\mathcal{F}_{i}$ describing the uncertainty of each direct measurement (i.e., measurement of $x_{i}$ ). We want to describe the resulting uncertainty class $\mathcal{F}$ for $y=f\left(x_{1}, \ldots, x_{n}\right)$. Two situations are possible:

- when measurement errors corresponding to different $x_{i}$ are independent random variables; the resulting class will be denoted by $f_{\text {indep }}\left(\mathcal{F}_{1}, \ldots, \mathcal{F}_{n}\right)$ and

- when measurement errors may be correlated; the resulting class will be denoted by $f_{\text {general }}\left(\mathcal{F}_{1}, \ldots, \mathcal{F}_{n}\right)$.

In this paper, we show that in some of these situations, for $y$, we can get an arbitrary closed convex class of probability distributions.

\section{TOWARDS A PRECISE FORMULATION OF THE PROBLEM}

\subsection{A CLASS $\mathcal{P}$ MUST BE CONVEX}

Indeed, let $P_{1}, P_{2} \in \mathcal{P}$. Since $P_{i} \in \mathcal{P}$, there exists a situation $s_{i}$ which is consistent with the measurements and in which probabilities are described by $P_{i}$. If we combine $N$ situations of type $s_{1}$ and $N$ situations of type $s_{2}$, then we get a new situation which is also consistent with measurements and for which $P=0.5 \cdot P_{1}+0.5 \cdot P_{2}$. Thus, $P \in \mathcal{P}$, and $\mathcal{P}$ is convex. 


\subsection{A CLASS $\mathcal{P}$ MUST BE CLOSED}

Indeed, if $P_{n} \rightarrow P$ (in some reasonable sense), and $P_{n} \in \mathcal{P}$, this means that all measurements are consistent with all $P_{n}$. Intuitively, the fact that $P$ is a limit of $P_{n}$ means that for an arbitrary accuracy, sufficiently large $P_{n}$ are, within this accuracy, indistinguishable from $P$. Since $P_{n}$ is consistent with all the measurements, it thus follows that $P$ should also be consistent with them.

\subsection{A NATURAL METRIC}

To complete our formalization, we must select an appropriate topology. Each measuring instrument has a range and an accuracy within this range. Thus, a natural topology means that the corresponding probability distributions are close within a given range. This can be formalized as follows (see, e.g., [7] for more detailed motivations): We say that a point $(x, p)$ is in the extended graph of the probability distribution $F(x)$ if $F(x-0) \leq p \leq F(x)$. Let $\varepsilon>0$. We say that the probability distributions $F(x)$ and $G(x)$ are $\varepsilon$-close if the following two properties hold:

- For every point $(x, p)$ from the extended graph of $F$ for which $|x| \leq 1 / \varepsilon$, there exists a point $(y, q)$ on the extended graph of $G$ for which

$$
|x-y| \leq \varepsilon \text { and }|p-q| \leq \varepsilon, \text { and }
$$

- vice versa.

For every two probability distributions $F$ and $G$, the distance $\rho(F, G)$ is defined as the smallest $\varepsilon>0$ for which $F$ and $G$ are $\varepsilon$-close.

When we consider only probability measures which are located on a given interval, the metric $\rho(F, G)$ becomes a Hausdorff distance between the (extended) graphs of the functions $F$ and $G$ with respect to sup-norm on the plane

$$
\left\|\left(x_{1}, p_{1}\right)-\left(x_{2}, p_{2}\right)\right\|=\max \left(\left|x_{1}-x_{2}\right|,\left|p_{1}-p_{2}\right|\right) .
$$

(In probability theory, this distance between probability measures is also known as Levý distance). Convergence in this metric is equivalent to socalled weak convergence of the distributions.

Definition 1. By an uncertainty class $\mathcal{F}$, we mean a convex closed class of probability distributions (closed w.r.t. the metric $\rho$ ).

Examples: For every probability distribution $F$, a one-element class $\mathcal{F}=\{F\}$ consisting of this distribution $F$ is an uncertainty class. For 
every interval $[a, b]$, the class $\mathcal{F}_{[a, b]}$ located on $[a, b]$, i.e., the class of all distributions $F$ for which $F(a-0)=0$ and $F(b)=1$, also forms an uncertainty class.

In view of this definition, we will define $f_{\text {indep }}\left(\mathcal{F}_{1}, \ldots, \mathcal{F}_{n}\right)$ as a convex closed hull of the set of all distributions which correspond to independent $F_{i} \in \mathcal{F}_{i}$, and $f_{\text {general }}\left(\mathcal{F}_{1}, \ldots, \mathcal{F}_{n}\right)$ as a convex closed hull of the set of all distributions which correspond to possibly dependent $F_{i} \in \mathcal{F}_{i}$.

\section{RESULTS}

\subsection{TRADITIONAL CASE: PROBABILITY DISTRIBUTIONS}

Traditionally, for each $x_{i}$, it is assumed that we know the probability distribution $F_{i}$, i.e., $\mathcal{F}_{i}=\left\{F_{i}\right\}$. If we know that these distributions are independent, then we have the complete description of the joint distribution for these $n$ variables $x_{1}, \ldots, x_{n}$. (Hence, $\mathcal{F}=\{\mathcal{F}\}$ is a one-element uncertainty class.)

The case of possible dependent probability distributions is described by a special theory called copula theory (see, e.g., [9]).

\subsection{INTERVAL CASE}

If for every $i$, the uncertainty of each variable $x_{i}, 1 \leq i \leq n$, is described by an interval $\left[\underline{x}_{i}, \bar{x}_{i}\right]$, i.e., by a class $\mathcal{F}_{\left[\underline{x}_{i}, \bar{x}_{i}\right]}$, then the uncertainty class $\mathcal{F}$ is also an interval (irrespective on whether the inputs are independent or not):

Proposition 1. Let $f\left(x_{1}, \ldots, x_{n}\right)$ be a continuous function of $n$ real variables, and let $\left.\left[\underline{x}_{1}, \bar{x}_{1}\right], \ldots, \underline{x}_{n}, \bar{x}_{n}\right]$ be $n$ intervals. Then,

$$
\begin{gathered}
f_{\text {indep }}\left(\mathcal{F}_{\left[\underline{x}_{1}, \bar{x}_{1}\right]}, \ldots, \mathcal{F}_{\left[\underline{x}_{n}, \bar{x}_{n}\right]}\right)= \\
f_{\text {general }}\left(\mathcal{F}_{\left[\underline{x}_{1}, \bar{x}_{1}\right]}, \ldots, \mathcal{F}_{\left[\underline{x}_{n}, \bar{x}_{n}\right]}\right)= \\
\mathcal{F}_{[\underline{[}, \bar{y}]},
\end{gathered}
$$

where

$$
\begin{gathered}
{[\underline{y}, \bar{y}]=f\left(\left[\underline{x}_{1}, \bar{x}_{1}\right], \ldots,\left[\underline{x}_{n}, \bar{x}_{n}\right]\right)=} \\
\left\{y=f\left(x_{1}, \ldots, x_{n}\right) \mid x_{1} \in\left[\underline{x}_{1}, \bar{x}_{1}\right], \ldots, x_{n} \in\left[\underline{x}_{n}, \bar{x}_{n}\right]\right\} .
\end{gathered}
$$

Comment. In our definitions, we defined both $f_{\text {indep }}$ and $f_{\text {general }}$ as convex hulls of the corresponding class of distributions. One can show that for $f_{\text {indep }}$, it is necessary to do that, while for $f_{\text {general }}$, the class is already convex, so taking a convex hull is not necessary. 


\subsection{MIXED CASE: INDEPENDENT PROBABILITY DISTRIBUTION AND INTERVAL}

Proposition 2. For every uncertainty class $\mathcal{F}$, and for every $\varepsilon>0$, there exists a probability distribution $F$, an interval $[a, b]$, and a continuous function $f\left(x_{1}, x_{2}\right)$ for which the uncertainty class $f_{\text {indep }}\left(\{F\}, \mathcal{F}_{[a, b]}\right)$ is $\varepsilon$-close to $\mathcal{F}$.

In other words, even in the simplest case when we only have two independent variables, one of which has a precisely known distribution, and the other is characterized by an interval of possible values, we can get an arbitrary uncertainty class. Thus, arbitrarily complicated uncertainty classes are not simply the result of a mathematical definition, they are practically possible.

Of course, this result does not necessarily means that we have to use arbitrarily complex uncertainty classes. We can restrict ourselves to a collection of simpler uncertainty classes such as p-bounds (see definition below; see also $[1,2,4]$ ). In this case, our result says that in some reallife situations, the description by these simple uncertainty classes will not be precise, it will only be an approximation.

Definition 2. An uncertainty class is called a p-bound if it consists of all distribution functions $F(x)$ for which $\underline{F}(x) \leq F(x) \leq \bar{F}(x)$ for all $x$, where $\underline{F}(x)$ and $\bar{F}(x)$ are given.

Comment. To better understand the difference between general uncertainty classes and p-bounds, let us give an example of an uncertainty class which is not a p-bound. Let $\underline{F}$ and $\bar{F}$ be two different distributions for which $\underline{F}(x)<\bar{F}(x)$ for all $x$. Then, the convex hull of these two distributions consists only of distributions of the type $\alpha \cdot \bar{F}(x)+(1-\alpha) \cdot \underline{F}(x)$. Elements of this set can be determined by a single parameter $\alpha$, and therefore, this set is 1-dimensional. In contrast, the corresponding p-bound contains an infinite-dimensional class of possible distributions. For example, to describe a finite-dimensional subclass of this class, with an arbitrary dimension $k$, we can select an arbitrary sequence of values $x_{(1)}<\ldots<x_{(k)}$ and the corresponding values $0<\alpha_{1}<\alpha_{2}<\ldots<\alpha_{k-1}<1$, and then define the distribution $F(x)$ from the p-bound as:

- $F(x)=\underline{F}(x)$ for $x<x_{(1)}$;

- $F(x)=\alpha_{i} \cdot \bar{F}(x)+\left(1-\alpha_{i}\right) \cdot \underline{F}(x)$ for $x_{(i)} \leq x<x_{(i+1)}$, and

- $F(x)=\bar{F}(x)$ for $x \geq x_{(k)}$. 


\subsection{PROOF OF PROPOSITION 1 (INDEPENDENT CASE)}

To prove this result, let us first show that the desired uncertainty class $f_{\text {indep }}\left(\mathcal{F}_{\left[\underline{x}_{1}, \bar{x}_{1}\right]}, \ldots, \mathcal{F}_{\left[\underline{x}_{n}, \bar{x}_{n}\right]}\right)$ is contained in $\mathcal{F}_{[\underline{y}, \bar{y}]}$. Indeed, if $P^{(n)}$ is a possible joint distribution, this means that all marginal distributions are located on the corresponding intervals $\left[\underline{x}_{i}, \bar{x}_{i}\right]$, and thus, only those combinations $\left(x_{1}, \ldots, x_{n}\right)$ are possible for which $x_{i} \in\left[\underline{x}_{i}, \bar{x}_{i}\right]$. For these combinations,

$$
\begin{gathered}
f\left(x_{1}, \ldots, x_{n}\right) \in[\underline{y}, \bar{y}]=f\left(\left[\underline{x}_{1}, \bar{x}_{1}\right], \ldots,\left[\underline{x}_{n}, \bar{x}_{n}\right]\right)= \\
\left\{y=f\left(x_{1}, \ldots, x_{n}\right) \mid x_{1} \in\left[\underline{x}_{1}, \bar{x}_{1}\right], \ldots, x_{n} \in\left[\underline{x}_{n}, \bar{x}_{n}\right]\right\},
\end{gathered}
$$

and therefore, every possible distribution $p^{(1)}$ is located on the interval $[\underline{y}, \bar{y}]$.

Let us now show that the desired uncertainty class coincides with the interval class $\mathcal{F}_{[\underline{y}, \bar{y}]}$. Since the desired uncertainty class is convex and closed, it is sufficient to prove that all extreme points of the interval class $\mathcal{F}_{[\underline{y}, \bar{y}]}$ (i.e., all distributions which cannot be described as non-trivial convex combinations of others) belong to the desired class; if we prove this result, then our conclusion will follow from the fact that the interval class coincides with the convex closed hull of all its extreme points.

Let us describe these extreme points. Any distribution which is not located in a single point is not an extreme point, because it can represented as a convex combination of distributions located at different points. Thus, the only extreme points are distributions which are located in a single point. If we take, for each $x_{i} \in\left[\underline{x}_{i}, \bar{x}_{i}\right]$, a distribution $F_{i}$ which is located with probability 1 in a point $x_{i}$, then the corresponding possible joint distribution $P^{(n)}=P_{1} \times \ldots \times P_{n}$ is located, with probability 1 , in a point $y=f\left(x_{1}, \ldots, x_{n}\right)$. Thus, for any point $y$ which can be represented as $f\left(x_{1}, \ldots, x_{n}\right)$ for some $x_{i} \in\left[\underline{x}_{i}, \bar{x}_{i}\right]$, i.e., for any point $y$ from the interval $[\underline{y}, \bar{y}]$ of all such points, the distribution which is located, with probability 1 , in the point $y$, is possible, and thus, belongs to the desired class.

An arbitrary distribution located on finitely many points from the interval $[\underline{y}, \bar{y}]$ can be represented as a convex combination of such onepoint distributions (with coefficients equal to the probabilities of the corresponding points), and therefore, also belongs to the desired class. Finally, an arbitrary distribution located on the interval $[y, \bar{y}]$ can be represented as a limit of distributions located on finitely many points (when these points grow denser); so, since the desired class is, by definition, closed (i.e., contains the limits of its elements), an arbitrary distribution 
located on the interval also belongs to the desired class. The result is proven.

\subsection{PROOF OF PROPOSITION 1 (GENERAL CASE)}

In the beginning of the proof for the independent case, we have proven, in effect, that the desired uncertainty class $f_{\text {general }}\left(\mathcal{F}_{\left[\underline{x}_{1}, \bar{x}_{1}\right]}, \ldots, \mathcal{F}_{\left[\underline{x}_{n}, \bar{x}_{n}\right]}\right)$ is contained in $\mathcal{F}_{[\underline{y}, \bar{y}]}$.

In the second part of that proof, we have shown that every distribution from the interval class $\mathcal{F}_{[y, \bar{y}]}$ belongs to the closed convex hull of all distributions which are possible under independence. Since distributions which are possible under independence form a subclass of possible distributions, we can this conclude that every distribution from the interval class $\mathcal{F}_{[\underline{y}, \bar{y}]}$ belongs to the closed convex hull of all possible distributions. Thus, every distribution from the interval class $\mathcal{F}_{[\underline{y}, \bar{y}]}$ belongs to the desired class.

These two results conclude the proof of Proposition 1.

\subsection{PROOF OF THE COMMENT AFTER PROPOSITION 1}

Let us first prove that if, in the definition of the class $f_{\text {indep }}\left(\mathcal{F}_{\left[\underline{x}_{1}, \bar{x}_{1}\right]}, \ldots, \mathcal{F}_{\left[\underline{x}_{n}, \bar{x}_{n}\right]}\right)$ we do not take the convex hull, then Proposition 1 will no longer hold. To be more precise, we will show an example of a distribution $P$ which is located on the interval $[\underline{y}, \bar{y}]$ but which is not possible (although, in accordance with Proposition 1 , it is a convex combination of possible distributions).

All we need is a single example, so let us consider a simple case when $n=2, f\left(x_{1}, x_{2}\right)=x_{1}+x_{2}$, and $\left[\underline{x}_{1}, \bar{x}_{1}\right]=\left[\underline{x}_{2}, \bar{x}_{2}\right]=[0,1]$. In this case, $[\underline{y}, \bar{y}]=f\left(\left[\underline{x}_{1}, \bar{x}_{1}\right],\left[\underline{x}_{2}, \bar{x}_{2}\right]\right)=[0,2]$. Let us show that the distribution $P$ which is located at 0 with probability 0.5 and at 2 with probability 0.5 is not possible under independence.

We will prove this impossibility by reduction to a contradiction. Let us assume that $P=F_{1} \times F_{2}$ for some possible distributions $F_{1}$ and $F_{2}$. Since we know that $y=x_{1}+x_{2}$ and $x_{i} \in[0,1]$, the only way to get $y=2$ is to get $x_{1}=x_{2}=1$. The fact that this value $y=2$ has a positive probability means that there is a positive probability that $x_{1}=1$ and a positive probability that $x_{2}=1$. Similar, the only way to get $y=x_{1}+x_{2}=0$ is to have $x_{1}=x_{2}=0$; the fact that this value $y=0$ has a positive probability means that there is a positive probability that $x_{1}=0$ and a positive probability that $x_{2}=0$. So, 
there is a positive probability that $x_{1}=0$ and a positive probability that $x_{2}=1$. Since $x_{1}$ and $x_{2}$ are independent, the probability that both $x_{1}=0$ and $x_{2}=1$ is also positive. So, with a positive probability, we should get $y=x_{1}+x_{2}=1$; however, in the original distribution $P$, the probability of $y=1$ is equal to 0 . This contradiction shows that our assumption was false, and $P$ cannot be represented as $F_{1} \times F_{2}$ (i.e., $P$ is not possible).

Let us now prove that in the definition of the class $f\left(\mathcal{F}_{1}, \ldots, \mathcal{F}_{n}\right)$, we do not need to require that we take a convex hull.

By definition of this class, it means that the class of all possible distributions $F^{(1)}$ is convex. Indeed, let $F^{(1)}$ and $G^{(1)}$ be possible distributions. Let us prove that their convex combination $\alpha \cdot F^{(1)}+(1-\alpha) \cdot G^{(1)}$ is also a possible distribution. Indeed, the fact that $F^{(1)}$ and $G^{(1)}$ are possible distributions means that they correspond to possible joint distributions $P^{(n)}$ and $Q^{(n)}$ accordingly. Therefore, the desired linear combination $\alpha \cdot F^{(1)}+(1-\alpha) \cdot G^{(1)}$ is also possible, because it corresponds to the joint distribution $\alpha \cdot P^{(n)}+(1-\alpha) \cdot Q^{(n)}$ which is easily proven to be possible too. The comment is proven.

\subsection{PROOF OF PROPOSITION 2}

In this proof, we will provide a step-by-step approximation of an uncertainty class $\mathcal{F}$ by an uncertainty class $f_{\text {indep }}\left(\{F\}, \mathcal{F}_{[a, b]}\right)$.

$1^{\circ}$. First, due to our definition of a metric $\rho$ on the set of all probability distributions, to guarantee that $\rho(F, G) \leq \delta$, we only need to consider the values $F(x)$ and $G(x)$ for $|x| \leq 1 / \delta$. Thus, if we pick, e.g., $\delta=\varepsilon / 4$, we only need to consider values of $F(x)$ for $x \in[-1 / \delta, 1 / \delta]$.

$2^{\circ}$. Due to the above restriction, the metric $\rho(F, G)$ is equal to the Hausdorff distance between the pieces of the (extended) graphs of the functions $F(x)$ and $G(x)$ which correspond to $x \in[-1 / \delta, 1 / \delta]$.

These graph pieces are contained in the set $S=[-1 / \delta, 1 / \delta] \times[0,1]$ which is closed and bounded and therefore, compact: for every $\varepsilon>0$, there is a finite set $S(\varepsilon) \subseteq S$ which is its $\varepsilon$-net, i.e., a set which has the property that for every $s \in S$, there is an $s^{\prime} \in S(\varepsilon)$ for which $\left\|s-s^{\prime}\right\| \leq \varepsilon$. If we recall the definition of a Hausdorff distance, we can conclude that $\rho(S, S(\varepsilon)) \leq \varepsilon$.

Moreover, one can show that for every closed subset $T \subseteq S$, the set $T(\varepsilon)$ formed by those points from $S(\varepsilon)$ which are $\varepsilon$-close to some points from $T$ is also $\varepsilon$-close to $T$. Thus, sets of this type $T(\varepsilon)$ form an $\varepsilon$-net for the set of all closed subsets of $S$. Each set of this type is a subset of a given finite set; therefore, this $\varepsilon$-net is also finite. 
Hence, we can repeat the same construction and conclude that every class of probability distributions can be approximated, within any given accuracy, by a finite class.

$4^{\circ}$. We have shown that every class of probability distributions can be approximated, within any given accuracy, by a finite class. Let $\left\{F_{1}, \ldots, F_{n}\right\}$ be such an approximation for the original class $\mathcal{F}$.

Since the original class $\mathcal{F}$ is an uncertainty class and hence is convex, we can therefore conclude that the convex hull of the finite class $\left\{F_{1}, \ldots, F_{n}\right\}$ is also sufficiently close to this original class $\mathcal{F}$. Moreover, we can conclude that an arbitrary class which is intermediate between the original finite class and its convex hull should be also $\delta$-close to $\mathcal{F}$. We will therefore proceed to construct a probability distribution $F$, an interval $[a, b]$, and a continuous function $f\left(x_{1}, x_{2}\right)$ for which the uncertainty class $f_{\text {indep }}\left(\{F\}, \mathcal{F}_{[a, b]}\right)$ is sufficiently close to such an intermediate class.

$5^{\circ}$. The functions $F_{i}(x)$ are not necessarily strictly monotonic; however, we can approximate them by strictly monotonic functions, e.g., by taking $(1-\delta) \cdot F_{i}(x)+\delta \cdot x$. In effect, what this transformation does is takes every horizontal line on a graph and makes it slightly increasing.

Similarly, these functions are not necessarily continuous, but we can make them continuous by an arbitrarily small (in the Hausdorff metric) modification of the (extended) graph; we can do that by applying, after the above-described modification of the function $F_{i}(x)$, a similar transformation to the corresponding "inverse" function $\varphi_{i}(p)$. Thus, we can approximate each function $F_{i}$ by a continuous strictly monotonic function. Since an approximation to an approximation is also an approximation to the original class, we can, for simplicity (and without losing generality), assume that the functions $F_{i}$ are already continuous and strictly monotonic.

$6^{\circ}$. We are now ready to describe the construction of the triple $(F,[a, b], f)$.

We will take, as $F$, the distribution of this uniform random variable (i.e., $F(x)=0$ for $x \leq 0, F(x)=x$ for $x \in[0,1]$, and $F(x)=1$ for $x \geq 1)$. As $[a, b]$, we take the interval $[0,1]$.

To construct the function $f$, we take into consideration that since we assume that each function $F_{i}(x)$ is continuous and strictly monotonic, each such function $F_{i}(x)$ can be represented as $\varphi_{i}(\xi)$, where $\xi$ is uniformly distributed on the interval $[0,1]$.

Now, as $f\left(x_{1}, x_{2}\right)$, we take a function which is defined as follows: 
- For $x_{2}=0$, we take $f\left(x_{1}, x_{2}\right)=\varphi_{1}\left(x_{1}\right)$.

- For $x_{1}=\frac{1}{n-1}$, we take $f\left(x_{1}, x_{2}\right)=\varphi_{2}\left(x_{1}\right)$.

- $\ldots$

- For $x_{1}=\frac{k-1}{n-1}$, where $k$ is an integer, we take $f\left(x_{1}, x_{2}\right)=\varphi_{k}\left(x_{1}\right)$.

- $\ldots$

- For $x_{1}=1$, we take $f\left(x_{1}, x_{2}\right)=\varphi_{n}\left(x_{1}\right)$.

- For all intermediate values, when

$$
\frac{k-1}{n-1}<x<\frac{k}{n-1},
$$

we find the unique $\alpha$ for which

$$
x=\alpha \cdot \frac{k}{n-1}+(1-\alpha) \cdot \frac{k-1}{n-1},
$$

and take, as $f\left(x_{2}, x_{2}\right)$, the value $f\left(x_{1}, x_{2}\right)=\varphi_{k, \alpha}\left(x_{1}\right)$, where $\varphi_{k, \alpha}(p)$ is an inverse function to the convex combination

$$
F_{k, \alpha}(x)=\alpha \cdot F_{k+1}(x)+(1-\alpha) \cdot F_{k}(x) .
$$

One can see that this piece-wise definition indeed leads to a continuous function $f\left(x_{1}, x_{2}\right)$ of two variables. Due to this construction, for each fixed $x_{2} \in[a, b]=[0,1]$, the resulting random variable $f\left(x_{1}, x_{2}\right)$ either coincides with one of the probability measures $F_{i}$, or with a convex combination of these measures. All measures $F_{i}$ are representable as $f\left(x_{1}, x_{2}\right)$ under deterministic values $x_{2}$ and are, therefore, possible under independence.

Also, whenever the distribution of $x_{2}$ is deterministic (i.e., located at a single point), the corresponding distribution for $y=f\left(x_{1}, x_{2}\right)$ belongs to the convex hull of the distributions $F_{1}, \ldots, F_{n}$.

$7^{\circ}$. An arbitrary distribution on the interval $[0,1]$ which is located in finitely many points $x_{2}^{(1)}, \ldots, x_{2}^{(k)}$, can be represented as a convex combination of deterministic distributions located at these points $x_{2}^{(j)}$ (with coefficients equal to the probabilities of these points).

Since $x_{1}$ and $x_{2}$ are assumed to be independent, for such a distribution on $x_{2}$, the resulting distribution for $y=f\left(x_{1}, x_{2}\right)$ is equal to the similar convex combination of the distributions $y^{(j)}=f\left(x_{1}, x_{2}^{(j)}\right)$. We already know that for each $j$, the distribution for $y^{(j)}$ belongs to the convex 
hull of $F_{i}$; therefore, the distribution function for $y$ (which is the convex combination of these distribution functions) also belongs to the convex hull of $F_{i}$.

$8^{\circ}$. An arbitrary distribution of the interval $[0,1]$ can be represented as a limit of distributions located at finite-point sets.

Thus, the corresponding distribution for $y=f\left(x_{1}, x_{2}\right)$ belongs to the closure of the convex hull of the distributions $F_{i}$. Since the convex hull of finitely many distributions is finite-dimensional, it is therefore closed, and so the distribution for $y$ actually belongs to the convex hull itself.

$9^{\circ}$. Let us summarize what we have proven:

- every distribution which is possible under independence belongs to the convex hull of the finite class $\left\{F_{1}, \ldots, F_{n}\right\}$, and

- all distributions $F_{i}$ are possible under independence.

Thus, the class $f_{\text {indep }}\left(\{F\}, \mathcal{F}_{[a, b]}\right)$ of all distributions which are possible under independence forms an intermediate class between the class $\left\{F_{1}, \ldots, F_{n}\right\}$ and its convex hull.

We already know that every such intermediate class is sufficiently close to this original class $\mathcal{F}$. Thus, the class $f_{\text {indep }}\left(\{F\}, \mathcal{F}_{[a, b]}\right)$ is sufficiently close to $\mathcal{F}$. The proposition is proven.

\section{Acknowledgments}

This work was supported in part by NASA under cooperative agreement NCC5209, by NSF grants No. DUE-9750858 and CDA-9522207, by United Space Alliance, grant No. NAS 9-20000 (PWO C0C67713A6), by the Future Aerospace Science and Technology Program (FAST) Center for Structural Integrity of Aerospace Systems, effort sponsored by the Air Force Office of Scientific Research, Air Force Materiel Command, USAF, under grants number F49620-95-1-0518 and F49620-00-1-0365, by the National Security Agency under Grant No. MDA904-98-1-0561 and MDA90498-1-0564, and by Grant No. W-00016 from the U.S.-Czech Science and Technology Joint Fund.

The authors are thankful to participants of SCAN'2000/Interval'2000 conference for valuable discussions.

\section{References}

[1] D. Berleant and H. Cheng, "A Software Tool for Automatically Verified Operations on Intervals and Probability Distributions", Reliable Computing, 1998, Vol. 4, No. 1, pp. 71-82.

[2] D. Berleant and C. Goodman-Strauss, "Bounding the Results of Arithmetic Operations on Random Variables of Unknown Depen- 
dency using Intervals", Reliable Computing, 1998, Vol. 4, No. 2, pp. $147-165$.

[3] H. Busemann, The geometry of geodesics, Academic Press, N.Y., 1955.

[4] S. Ferson and L. Ginzburg, "Hybrid arithmetic", In: B. M. Ayyub (ed.), Proceedings of the ISUMA-NAFIPS'95, IEEE Computer Society Press, Los Alamitos, CA, 1995, pp. 619-623.

[5] K. Ito (ed.), Encyclopedic dictionary of mathematics, MIT Press, Cambridge, MA, 1993.

[6] R. B. Kearfott and V. Kreinovich (eds.), Applications of Interval Computations, Kluwer, Dordrecht, 1996.

[7] V. Kreinovich, S. Ferson, L. Ginzburg, H. Schulte, M. R. Barry, and H. T. Nguyen, "From Interval Methods of Representing Uncertainty To A General Description of Uncertainty", In: H. Mohanty and C. Baral (eds.), Trends in Information Technology, Proceedings of the International Conference on Information Technology ICIT'99, Bhubaneswar, India, December 20-22, 1999, Tata McGraw-Hill, New Delhi, 2000, pp. 161-166.

[8] P. Lévy, Théorie de l'addition des variables aléatoires, GauthierVillars, Paris, 1937.

[9] R. B. Nelsen, An introduction to copulas, Springer-Verlag, New York, 1999.

[10] A. N. Shiryaev, Probability, Springer-Verlag, N.Y., 1984. 